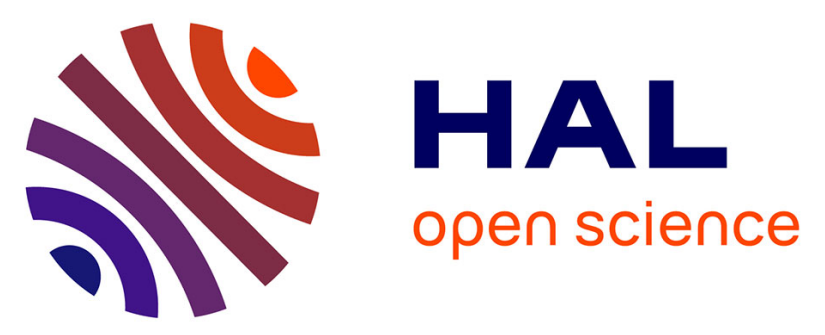

\title{
Functionalization of electrostatic nonlinearities to overcome mode aliasing limitations in the sensitivity of mass microsensors based on energy localization
}

Toky Harrison Rabenimanana, Vincent Walter, Najib Kacem, Patrice Le Moal, Gilles Bourbon, Joseph Lardies

\section{To cite this version:}

Toky Harrison Rabenimanana, Vincent Walter, Najib Kacem, Patrice Le Moal, Gilles Bourbon, et al.. Functionalization of electrostatic nonlinearities to overcome mode aliasing limitations in the sensitivity of mass microsensors based on energy localization. Applied Physics Letters, 2020, 117 (3), pp.033502 (6). hal-02993933

\section{HAL Id: hal-02993933 \\ https://hal.science/hal-02993933}

Submitted on 7 Nov 2020

HAL is a multi-disciplinary open access archive for the deposit and dissemination of scientific research documents, whether they are published or not. The documents may come from teaching and research institutions in France or abroad, or from public or private research centers.
L'archive ouverte pluridisciplinaire $\mathbf{H A L}$, est destinée au dépôt et à la diffusion de documents scientifiques de niveau recherche, publiés ou non, émanant des établissements d'enseignement et de recherche français ou étrangers, des laboratoires publics ou privés. 


\title{
Functionalization of electrostatic nonlinearities to overcome mode aliasing limitations in the sensitivity of mass microsensors based on energy localization
}

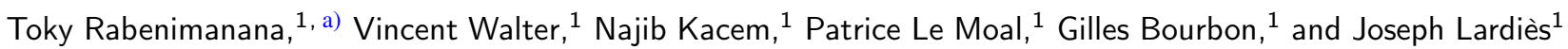 \\ Univ. Bourgogne Franche-Comté, FEMTO-ST Institute, CNRS/UFC/ENSMM/UTBM, Department of Applied Mechanics, \\ Besançon 25000, France
}

(Dated: 30 June 2020)

\begin{abstract}
In order to overcome mode aliasing limitation in linear mode-localized sensors, we demonstrate in this letter both theoretically and experimentally how to tune the electrostatic nonlinearity and exceed the ultimate sensitivity reachable in the linear regime. A mass sensor composed of two coupled micro-cantilevers with different lengths is considered and the results show that the sensitivity can be significantly enhanced up to $67 \%$. By performing experiments on the fabricated device and depositing the mass perturbation with a focused ion beam, the same phenomenon has been observed. These promising results open the way towards ultrasensitive multimodal microsensors with functionalized nonlinearities.
\end{abstract}

The ability of detecting a small mass with MEMS sensor constitutes a major interest for biomedical and chemical applications. The common principle used by mass sensors consists of a detection based on a frequency shift of a single resonator ${ }^{1-4}$. In order to bring enhancements to these devices, other sensing methods like mode localization ${ }^{5,6}$ have been investigated. It uses the change in the vibration mode of weakly coupled resonators to detect a small perturbation. With this method, the normalized sensitivity of the sensor is improved $^{7}$ and the effects of the ambient condition changes are reduced by the common mode rejection ${ }^{8}$. Most of mode localized mass sensors use two coupled resonators ${ }^{9-11}$ but others use more than two ${ }^{12,13}$. It also concerns other applications like stiffness sensing ${ }^{14,15}$ and acceleration sensing ${ }^{16}$. So far, all of these sensors use coupled linear resonators and the way to increase the sensitivity of the device is to reduce the ratio between the stiffness of the coupling and that of the resonator. However, the more the coupling decreases, the closer the frequencies of each mode are to each other. Due to the energy loss that defines the quality factor of the device, this coupling ratio has a minimum value that prevents the mode aliasing ${ }^{17}$. This corresponds to the minimum coupling that allows the separation between the peaks of each vibration mode. Thus, the sensitivity in the linear regime is limited. In order to overcome this limitation, we propose in this letter to tune the nonlinearities to enhance the sensitivity without changing the coupling. Devices with coupled nonlinear resonators have already been studied ${ }^{18-22}$ and proposed for energy harvesting to increase the frequency bandwidth ${ }^{23,24}$. Nevertheless, nonlinearity functionalization and its benefits for mass sensing utilizing mode localization have not yet been deeply addressed and experimentally demonstrated with respect to the ultimate sensitivity in the linear regime. In case of electrostatically actuated device, nonlinearities can be introduced by driving the device at high vibration amplitude, and the functionalization of these nonlinearities is their implementation and tuning in order to improve the device performances. So, we consider

\footnotetext{
a)Electronic mail: toky.rabenimanana@femto-st.fr
}

two weakly coupled cantilevers with electrostatic actuation in this study. An analytical analysis of its dynamic behavior is briefly presented, and the problem is solved with the method of multiple scale ${ }^{25}$, which has already been used for other nonlinear systems ${ }^{26,27}$. Simulations are then performed to compare the results between the linear case involving low vibration amplitudes and the nonlinear case involving high vibration amplitudes, when a same mass perturbation is added on the sensor. Furthermore, experiments are also conducted to validate the theoretical trends.

The considered device is composed of two mechanically coupled polysilicon cantilevers with different lengths, as described in Fig. 1, and its dimensions are given in Table. I. The quality factor used for the simulation was determined from experimental measurements and its value is $Q=770$. From this, we set the coupling ratio, which is equal to the ratio between the apparent stiffness of the coupling beam and the cantilever, to be near the limit of the aliasing mode. This minimum coupling ratio has been identified in the case of a discrete coupled model $^{17}$ as the inverse of the quality factor, i.e. $1 / Q=0.13 \%$. For the actuation, the short microbeam is actuated with a combined AC/DC voltage. The design of the device has already been studied on the basis of a theoretical linear approach ${ }^{28}$.

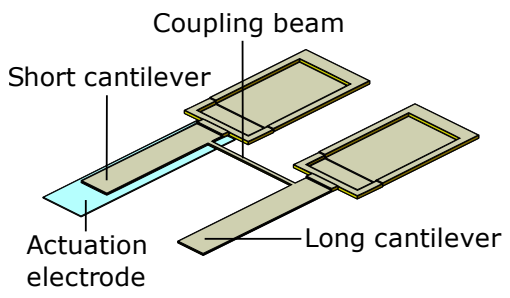

FIG. 1. The device composed of two mechanically coupled cantilevers with an actuation electrode under the short cantilever

Mode localized sensors require identical resonators, but the microfabrication technologies induce manufacturing defects preventing from getting this. With two microbeams of different lengths, we can counterbalance the manufacturing defects and the length difference by using the softening effect of the DC voltage ${ }^{29}$. Thus, we can reduce the apparent stiffness of 
TABLE I. Dimensions of the device

\begin{tabular}{cc}
\hline \hline Dimension & Value \\
\hline Lengths of the cantilevers & $98 \mu \mathrm{m}$ and $100 \mu \mathrm{m}$ \\
Width of the cantilevers & $19 \mu \mathrm{m}$ \\
Thickness of the cantilevers & $1.3 \mu \mathrm{m}$ \\
Length of the coupling beam & $65 \mu \mathrm{m}$ \\
Width of the coupling beam & $3 \mu \mathrm{m}$ \\
Position of the coupling from the fixed end & $4.6 \mu \mathrm{m}$ \\
\hline \hline
\end{tabular}

the short cantilever and equilibrate the system when the two cantilevers have the same resonant frequency. According to a previous work ${ }^{20,28}$, the device can be modeled by two coupled Euler-Bernoulli beams, and the equation describing the bending vibration of this system after discretization is similar to the equation of a nonlinear resonator (Short cantilever 1) coupled with a linear resonator (Long cantilever 2). A second order multiple scale method is used to solve the problem and the equation of the system is rewritten as follows

$$
\begin{aligned}
& \ddot{a}_{1}+\omega_{1}^{2} a_{1}=-2 \varepsilon^{2} \mu_{1} \dot{a}_{1}-\varepsilon^{2} k\left(\gamma_{1} a_{1}-\gamma_{2} a_{2}\right) \\
&-\varepsilon \alpha_{2} a_{1}{ }^{2}-\varepsilon^{2} \alpha_{3} a_{1}^{3}+\varepsilon^{2} f_{1} \cos (\Omega t) \\
& \ddot{a}_{2}+\omega_{2}^{2} a_{2}=-2 \varepsilon^{2} \mu_{2} \dot{a}_{2}-\varepsilon^{2} k\left(\gamma_{3} a_{2}-\gamma_{4} a_{1}\right)
\end{aligned}
$$

where dots denote the partial differentiation with respect to the time, $a_{i}$ are the deflection of the cantilever $i(i=1,2), \omega_{i}$ are the eigenfrequency, $2 \varepsilon^{2} \mu_{i}$ are the damping coefficient, $\varepsilon^{2} k$ is the coupling, $\varepsilon \alpha_{2}$ is the coefficient for the quadratic nonlinearity, $\varepsilon^{2} \alpha_{3}$ is the coefficient for the cubic nonlinearity, $\varepsilon^{2} f_{1}$ is the amplitude of the excitation force, $\Omega$ is the excitation frequency and $\varepsilon$ is a low-value parameter $(\varepsilon \ll 1)$. To proceed, we introduce different scales of time $T_{n}$ depending on $\varepsilon$ and defined by

$$
T_{n}=\varepsilon^{n} t \quad(n=1,2)
$$

As the frequency $\Omega$ of the harmonic load and the frequencies $\omega_{1}$ and $\omega_{2}$ of the two resonators are very close, we can also set the following relationships

$$
\begin{aligned}
& \Omega=\omega_{1}+\varepsilon^{2} \sigma_{1}, \\
& \omega_{1}=\omega_{2}+\varepsilon^{2} \sigma_{2}
\end{aligned}
$$

For more details about the solving method, readers can see supplementary material. This leads to the two following equations

$$
\begin{gathered}
{\left[\left(3 \alpha_{3}-\frac{10}{3} \frac{\alpha_{2}^{2}}{\omega_{1}^{2}}\right) u_{1}^{3}+\left(\left(-2 \omega_{1} \sigma_{1}+k \gamma_{1}\right)\right.\right.} \\
\left.\left.-k \gamma_{2} \frac{\left[-2 \omega_{2}\left(\sigma_{1}+\sigma_{2}\right)+k \gamma_{3}\right] k \gamma_{4}}{\left[-2 \omega_{2}\left(\sigma_{1}+\sigma_{2}\right)+k \gamma_{3}\right]^{2}+\left(2 \omega_{2} \mu_{2}\right)^{2}}\right) u_{1}\right]^{2} \\
+\left[\left(k \gamma_{2} \frac{2 \omega_{2} \mu_{2} k \gamma_{4}}{\left[-2 \omega_{2}\left(\sigma_{1}+\sigma_{2}\right)+k \gamma_{3}\right]^{2}+\left(2 \omega_{2} \mu_{2}\right)^{2}}\right.\right. \\
\left.\left.+2 \omega_{1} \mu_{1}\right) u_{1}\right]^{2}-\frac{1}{4} f_{1}^{2}=0
\end{gathered}
$$

$$
u_{2}=\frac{k \gamma_{4}}{\sqrt{\left[-2 \omega_{2}\left(\sigma_{1}+\sigma_{2}\right)+k \gamma_{3}\right]^{2}+\left(2 \omega_{2} \mu_{2}\right)^{2}}} u_{1}
$$

where the amplitude of $a_{1}$ and $a_{2}$ are respectively equal to $2 u_{1}$ and $2 u_{2}$. Eq. 5 is a sixth order equation which can be reduced to a cubic equation after a variable change. Solving this equation gives us the value of $u_{1}$ for each frequency, thus the frequency response of the short cantilever can be plotted. In nonlinear regime, Eq. 5 can give three real solutions that is why multivalued region is formed in the amplitude curve, while for the long cantilever, we just use Eq. 6 .

In order to investigate the effects of electrostatic nonlinearities on the mode localization, we first review its principle. When two identical resonators are weakly coupled, we have two modes of vibration corresponding to the symmetric and the antisymmetric modes. When a small mass perturbation is added only on the first resonator, its resonant frequency decreases, so it vibrates more in the first mode of the coupled system, i.e. at the lowest frequency. The second resonator vibrates more in the second mode of the coupled system. The vibration of the system thus becomes localized on the first resonator for the first mode, and on the second one for the second mode. Because only the first resonator is actuated to generate the vibration of the system, the mode localization will be more marked on mode 1 , so the ratio of vibration amplitudes on mode 1 will be used as output metrics to measure the mass perturbation. If we now introduce electrostatic nonlinearities in the first resonator, its resonant frequency becomes amplitudedependent. More precisely, the nonlinearity bends the frequency response graph to the left when the vibration amplitude increases, so the resonant frequency decreases. This appears when we only have electrostatic nonlinearity, leading to softening behavior. We have on the one hand, the mass perturbation decreasing the resonance frequency and increasing the vibration amplitude of the first resonator on the first mode, and on the other hand, the electrostatic nonlinearities further decreasing the resonance frequency when the vibration amplitude increases. As the added mass and the nonlinearity both lead to a decrease in the resonant frequency, we should have a more localized vibration than in coupled linear resonators, for the same mass perturbation. Thus, the sensitivity of the sensor can be improved.

In order to highlight the benefits of electrostatic nonlinearities on mass sensing, several simulations are performed. We first consider the linear case and find the DC voltage balancing the system. With a capacitance gap of $1.24 \mu \mathrm{m}$, the theoretical frequency response of the device at the balanced state is shown in Fig. 2(a), where the actuation is $v_{A C}=6 \mathrm{mV}$ and $V_{D C}=6.82 \mathrm{~V}$. Given that the DC voltage is not very close to the pull-in voltage that is theoretically evaluated and experimentally measured around $14 \mathrm{~V}$, the small $\mathrm{AC}$ voltage allows us to ensure that vibrations are linear. If we add a discrete mass of $10 \mathrm{pg}$ at the end of the short cantilever, the vibration becomes localized as shown in Fig.2(b). On the first mode, the ratio between the amplitude of the short cantilever and the long cantilever which is initially close to 1 becomes $W_{1} / W_{2}=2.2$ (measured on the circled dots). Afterwards, we perform simulations with high AC voltage. The result is 
shown in Fig. 2(c) in which the actuation is $v_{A C}=25 \mathrm{mV}$ and $V_{D C}=6.74 \mathrm{~V}$. We notice that a lower DC voltage is required because of the additional softening effect of the electrostatic nonlinearities ${ }^{20}$. With the same added mass of $10 \mathrm{pg}$, the result in Fig. 2(d) shows that the vibration on the first mode is more localized than in the linear case. The amplitude ratio on the first mode becomes $W_{1} / W_{2}=3$. We notice that the short cantilever that is actuated shows a softening behavior due to the high vibration amplitude. As the long cantilever is connected to the shorter one, there is a bifurcation topology transfer ${ }^{30}$ between the two resonators when the sensor is driven beyond its critical amplitude. Consequently, the long cantilever also shows a softening behavior even if it is not electrostatically actuated.
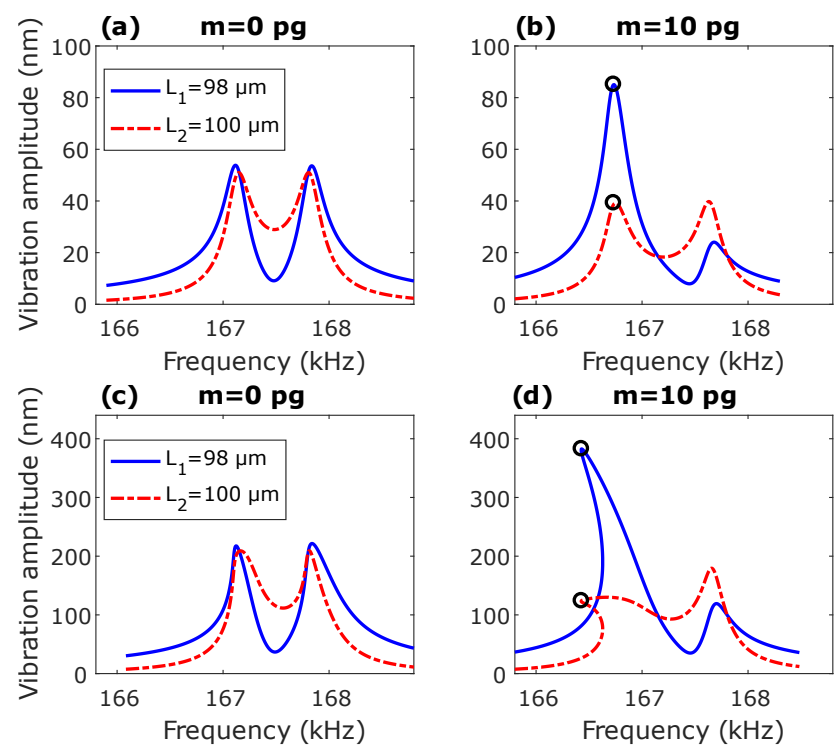

FIG. 2. Theoretical frequency responses of the device before and after adding a discrete point mass of $10 \mathrm{pg}$ at the end of the short cantilever. (a) and (b): $v_{A C}=6 \mathrm{mV}$ and $V_{D C}=6.82 \mathrm{~V}$. (c) and (d): $v_{A C}=25 \mathrm{mV}$ and $V_{D C}=6.74 \mathrm{~V}$.

To study the sensor sensitivity, we perform simulations over a mass range from $0 \mathrm{pg}$ to $10 \mathrm{pg}$ for different values of the $\mathrm{AC}$ voltage. For an $\mathrm{AC}$ voltage lower than $6 \mathrm{mV}$, the results in Fig. 3(a) show that the same amplitude ratio is obtained for a given added mass reflecting the linear regime. For higher AC voltage values, the amplitude ratio clearly increases for a given added mass. Fig. 3(a) also shows that the amplitude ratio is still a linear function of the added mass, therefore the sensitivity of the device remains constant over the mass range for a given AC voltage. Then, the evolution of the sensitivity is plotted as a function of the AC voltage in Fig. 3(b). As the sensitivity also depends on the location of the added mass, Fig. 3(b) incorporates the influence of this parameter as the normalized position $x_{m}$ of the mass (ratio between its position from the fixed end and the length of the short cantilever). For an added mass near the fixed end of the cantilever, the sensitivity is almost equal to zero, so we start the analysis from $x_{m}=0.6$. The sensitivity increases as we move away towards the free end (when $x_{m}$ increases). Therefore, the func- tionalized surface of the sensor to receive the elements to be weighed should be as close as possible to the free end of the cantilever (i.e. $x_{m}=1$ ). In the case of the linear regime below $6 \mathrm{mV}$ AC voltage and an added mass at the free end of the cantilever, the sensitivity is around $0.12 / p g$. For higher AC voltages, the sensitivity increases in the nonlinear regime to reach $0.20 / p g$ for $v_{A C}=25 \mathrm{mV}$. Thus, by introducing electrostatic nonlinearities, the sensitivity of the device has been increased by about $67 \%$. As the coupling ratio is at its optimal value in terms of sensitivity in the linear regime (i.e. the lowest value allowed by the aliasing mode phenomenon), it can be considered that this limit has been overcome by the implementation of electrostatic nonlinearities.

(a)

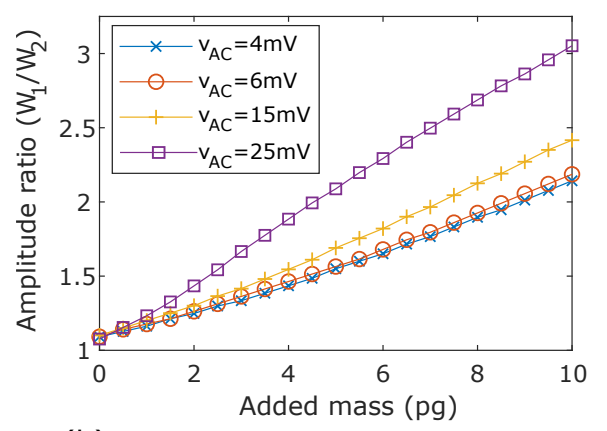

(b)

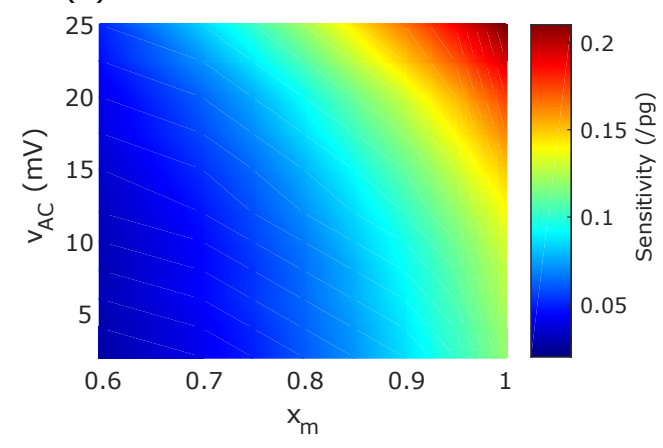

FIG. 3. (a) Variation of the amplitude ratio on the first mode as a function of the mass added at the end of the short cantilever and the $\mathrm{AC}$ voltage. (b) Variation of the sensitivity as a function of the AC voltage and the normalized position $x_{m}$ of the mass

We also notice that the vibration amplitude of the nonlinear case is almost four times higher than those of the linear case. As the mode localization uses a detection based on the amplitude measurement, the minimal detectable mass depends directly on the minimal detectable amplitude. Thus, using the nonlinearities with high vibration amplitude also enhances the mass resolution of the device.

In support of the theoretical results, experimental investigation is also conducted. The device (Fig. 4(a)) is fabricated with the Multi-User MEMS Processes and the mass perturbation is added by using focused ion beam deposition of platinum on the first cantilever (Fig. 4(b)). The device is first placed in a vacuum chamber where the pressure is around $0.50 \mathrm{mbar}$, and the actuation voltage is applied with a micro probe. To measure the vibration amplitude at the end of each 

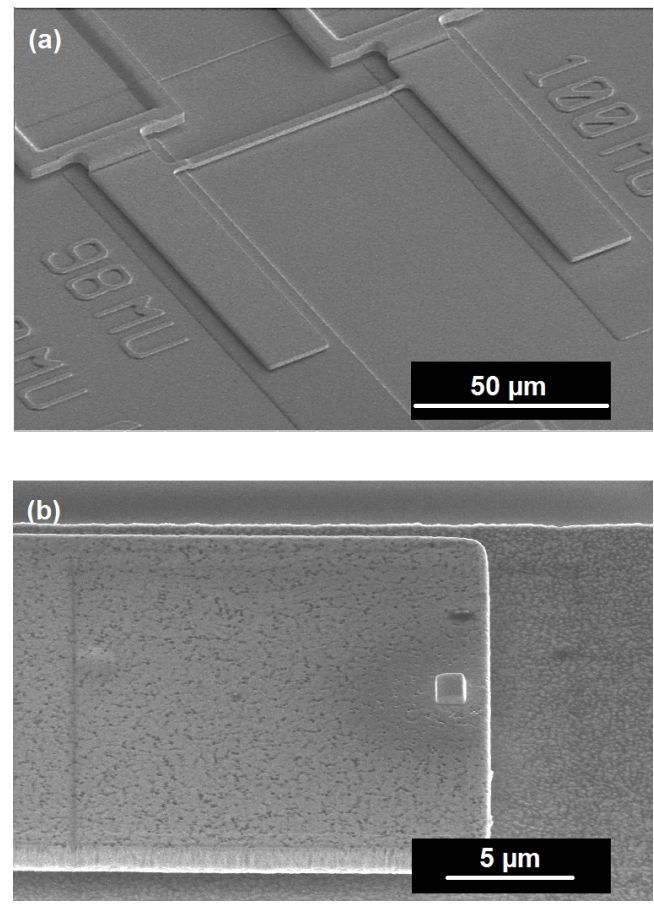

FIG. 4. (a): SEM image of the device. (b): SEM image of the deposited mass at the end of the short cantilever

cantilever, we first use a laser Doppler vibrometer in order to experimentally prove the concept, but a piezoresistive readout can in an applicative framework be implemented on this kind of mass sensor. Without the mass perturbation, the experimental frequency responses of the device are shown in Fig. 5. Fig. 5(a) corresponds to the linear case where $v_{A C}=6 \mathrm{mV}$ and $V_{D C}=6.82 \mathrm{~V}$, and Fig. 5(c) to the nonlinear case where $v_{A C}=25 \mathrm{mV}$ and $V_{D C}=6.74 \mathrm{~V}$. Once we get these results, we deposit a mass around $10 \mathrm{pg}$ on the short cantilever and plot again the frequency responses with the same actuation voltages used previously. By measuring the amplitude ratio of the first mode for the linear case, we found in Fig. 5(b) $W_{1} / W_{2}=2.3$ (measured on the circled dots). With a higher $\mathrm{AC}$ voltage where the vibrations are nonlinear, Fig. 5(d) gives an amplitude ratio equal to $W_{1} / W_{2}=3.9$. As shown by the simulation, the vibration of the sensor becomes more localized when the electrostatic nonlinearities are introduced at high vibration amplitudes.

We note a slight difference between experimental and theoretical amplitude ratios in the nonlinear regime. It could be due to the fact that we have some uncertainties about parameters used in the model. The first parameter concerns the quality factor which can change the vibration amplitude and the effect of electrostatic nonlinearities. As the pressure inside the vacuum chamber can change slightly, the quality factor before and after depositing the mass may not be the same. The second parameter concerns the gap which can change the cubic nonlinearity, the effect of electrostatic nonlinearities, and thus the sensitivity in the nonlinear regime. In addition, the analytical model may have limitations. A linear damping force is used while other authors proposed a model with a quadratic
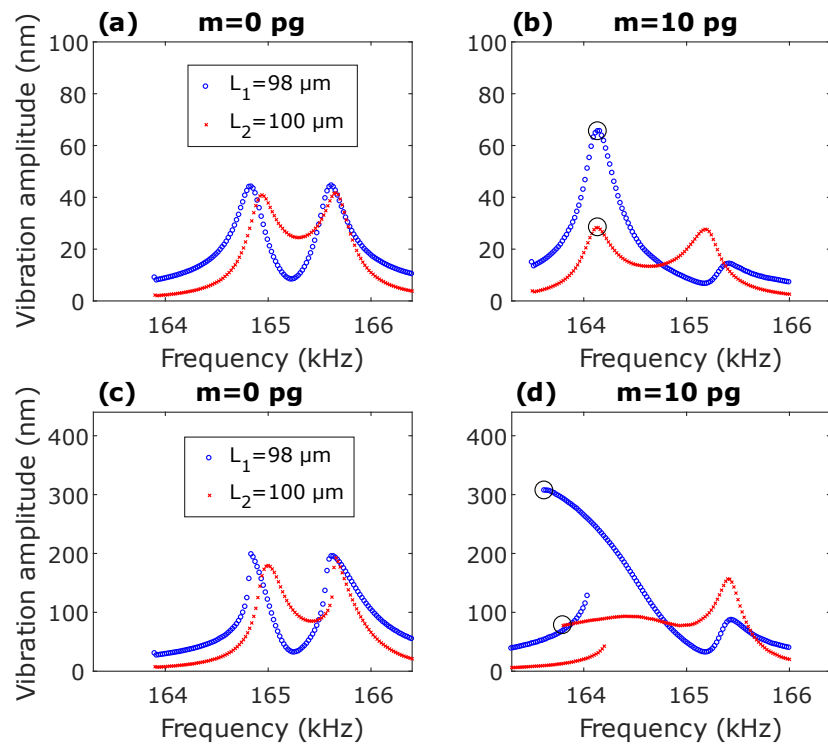

(d)

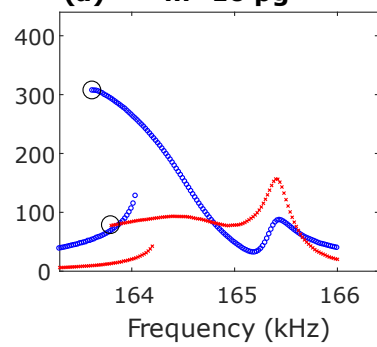

FIG. 5. Experimental frequency responses of the device before and after adding a mass of $10 \mathrm{pg}$ at the end of the short cantilever. (a) and (b): $v_{A C}=6 \mathrm{mV}$ and $V_{D C}=6.82 \mathrm{~V}$. (c) and (d): $v_{A C}=25 \mathrm{mV}$ and $V_{D C}=6.74 \mathrm{~V}$.

damping coefficient ${ }^{31}$. A third order Taylor series is used to expand the electrostatic force in the model, but this method may not be very accurate with very high vibration amplitudes. We also have some uncertainties about the actual deposited mass. As the device is more sensitive when it is operated in the nonlinear regime, the influence of these uncertainties becomes more important.

One also notes a small difference between the frequencies of the short and the long cantilevers at peak amplitude, especially in Fig. 5(d) where we have the jump phenomenon. It is due to the fact that the amplitude measurement on each cantilever is not simultaneous. After experimental verification, we do not have this difference if we just measure the vibration around the first mode. The two cantilevers are also connected, so they should have the same behavior.

In conclusion, the functionalization of the electrostatic nonlinearities to improve the sensitivity of a mode-localized mass sensor without changing the coupling is demonstrated in this letter. By using an analytical model, the comparison between the linear and nonlinear frequency responses of the sensor shows that we have more localized vibrations on the nonlinear case when the same mass is added on the same device. By using a high $\mathrm{AC}$ voltage involving high vibration amplitude, a nonlinearity is introduced and easily tuned, which results in a higher amplitude ratio. Following this, the sensitivity limitation due to mode aliasing is overcome with a significant improvement of up to $67 \%$. In addition, the mass resolution of the device is improved, due to the use of a higher vibration amplitude accurately detectable. After experimental measurements, we have also obtained the same improvement in the device response, which confirms the simulations. For future works, several devices can be considered in order to determine 
the accuracy of the mass sensing.

The data that supports the findings of this study are available within the article and its supplementary material.

See supplementary material for more details about the solving of Eq. 1 and 2.

\section{ACKNOWLEDGMENTS}

This project was supported by the Bourgogne-FrancheComté region and the EUR EIPHI program (ANR 17-EURE0002).

${ }^{1}$ A. Gupta, D. Akin, and R. Bashir, Applied Physics Letters 84, 1976 (2004).

${ }^{2}$ K. Ekinci, X. Huang, and M. Roukes, Applied Physics Letters 84, 4469 (2004).

${ }^{3}$ S. Dohn, R. Sandberg, W. Svendsen, and A. Boisen, Applied Physics Letters 86, 233501 (2005).

${ }^{4}$ Z. J. Davis and A. Boisen, Applied Physics Letters 87, 013102 (2005).

${ }^{5}$ P. W. Anderson, Physical review 109, 1492 (1958).

${ }^{6}$ O. O. Bendiksen, AIAA journal 25, 1241 (1987).

${ }^{7}$ C. Zhao, M. H. Montaseri, G. S. Wood, S. H. Pu, A. A. Seshia, and M. Kraft, Sensors and Actuators A: Physical 249, 93 (2016).

${ }^{8} \mathrm{P}$. Thiruvenkatanathan, J. Yan, and A. A. Seshia, in 2009 IEEE International Frequency Control Symposium Joint with the 22nd European Frequency and Time forum (IEEE, 2009) pp. 358-363.

${ }^{9}$ M. Spletzer, A. Raman, A. Q. Wu, X. Xu, and R. Reifenberger, Applied Physics Letters 88, 254102 (2006).

${ }^{10}$ P. Thiruvenkatanathan, J. Yan, J. Woodhouse, A. Aziz, and A. Seshia, Applied Physics Letters 96, 081913 (2010).

${ }^{11}$ G. S. Wood, C. Zhao, S. H. Pu, S. A. Boden, I. Sari, and M. Kraft, Microelectronic Engineering 159, 169 (2016).

${ }^{12}$ M. Spletzer, A. Raman, H. Sumali, and J. P. Sullivan, Applied Physics Letters 92, 114102 (2008).
${ }^{13}$ D. F. Wang, X. Li, X. Yang, T. Ikehara, and R. Maeda, Journal of Micromechanics and Microengineering 25, 095017 (2015).

${ }^{14}$ M. Manav, G. Reynen, M. Sharma, E. Cretu, and A. Phani, Journal of Micromechanics and Microengineering 24, 055005 (2014).

${ }^{15}$ H. Zhang, H. Chang, and W. Yuan, Microsystems \& nanoengineering 3, 17023 (2017).

${ }^{16}$ H. Zhang, B. Li, W. Yuan, M. Kraft, and H. Chang, Journal of microelectromechanical systems 25, 286 (2016).

${ }^{17}$ P. Thiruvenkatanathan, J. Woodhouse, J. Yan, and A. A. Seshia, Journal of Applied Physics 109, 104903 (2011).

${ }^{18}$ R. Lifshitz and M. Cross, Physical Review B 67, 134302 (2003).

${ }^{19}$ R. Karabalin, M. Cross, and M. Roukes, Physical Review B 79, 165309 (2009).

${ }^{20}$ T. Rabenimanana, V. Walter, N. Kacem, P. L. Moal, and J. Lardiès, in Multidisciplinary Digital Publishing Institute Proceedings, Vol. 2 (2018) p. 1084.

${ }^{21}$ S. Baguet, V.-N. Nguyen, C. Grenat, C.-H. Lamarque, and R. Dufour, Nonlinear Dynamics 95, 1203 (2019).

${ }^{22}$ M. Lyu, J. Zhao, N. Kacem, P. Liu, B. Tang, Z. Xiong, H. Wang, and Y. Huang, International Journal of Non-Linear Mechanics, 103455 (2020).

${ }^{23}$ L. Xiong, L. Tang, and B. R. Mace, Applied Physics Letters 108, 203901 (2016).

${ }^{24}$ W. Yang and S. Towfighian, Journal of Sound and Vibration 446, 129 (2019).

${ }^{25}$ A. H. Nayfeh and D. T. Mook, Nonlinear oscillations (John Wiley \& Sons, 2008).

${ }^{26} \mathrm{P}$. Alevras, S. Theodossiades, and H. Rahnejat, Applied Physics Letters 110, 233901 (2017)

${ }^{27}$ R. Liu, L. Wang, and J. Zhao, Applied Physics Letters 113, 211901 (2018).

${ }^{28}$ T. Rabenimanana, V. Walter, N. Kacem, P. Le Moal, G. Bourbon, and J. Lardies, Sensors and Actuators A: Physical 295, 643 (2019).

${ }^{29}$ V. Walter, G. Bourbon, P. Le Moal, N. Kacem, and J. Lardies, Procedia Engineering 168, 1488 (2016).

${ }^{30}$ D. Bitar, N. Kacem, and N. Bouhaddi, International Journal of Mechanical Sciences 127, 130 (2017).

${ }^{31}$ J. Juillard, A. Mostafa, and P. M. Ferreira, IEEE Transactions on Ultrasonics, Ferroelectrics, and Frequency Control 66, 1950 (2019). 